\title{
Corrigendum: Does Cognitive Behavior Therapy for psychosis (CBTp) show a sustainable effect on delusions? A meta-analysis
}

\author{
Stephanie Mehl ${ }^{1,2 *}$, Dirk Werner ${ }^{3}$ and Tania M. Lincoln ${ }^{4}$ \\ ${ }^{1}$ Department of Psychiatry and Psychotherapy, Philipps-University Marburg, Marburg, Germany, ${ }^{2}$ Department of Health and \\ Social Work, Frankfurt University of Applied Science, Frankfurt, Germany, ${ }^{3}$ Department of Psychological Methods and \\ Statistics, University of Hamburg, Hamburg, Germany, ${ }^{4}$ Department of Clinical Psychology and Psychotherapy, University of \\ Hamburg, Hamburg, Germany
}

Keywords: CBT, CBTp, delusions, paranoia, follow-up

\section{A Corrigendum on}

Does Cognitive Behavior Therapy for psychosis (CBTp) show a sustainable effect on delusions? A meta-analysis

by Mehl, S., Werner, D., and Lincoln, T. M. (2015). Front. Psychol. 6:1450. doi: 10.3389/fpsyg.2015.01450

In the original article, there were two errors. First, the effect size of one study (Turkington et al., 2006) was incorrect and this error resulted in an incorrect mean effect size for the comparison between Cognitive Behavior Therapy for psychosis (CBTp) and Treatment as Usual (TAU) at follow up. Further, there were errors in the classification of several studies as blind versus non-blind. These errors result in several corrections that are described, as follows.

First, the reported results and the discussion of the results in the Abstract section are incorrect. A correction has been made to the Abstract in the description of the results and the discussion.

"Cognitive Behavior Therapy for psychosis (CBTp) is an effective treatment resulting in small to medium effect sizes with regard to changes in positive symptoms and psychopathology. As a consequence, CBTp is recommended by national guidelines for all patients with schizophrenia. However, although CBTp was originally developed as a means to improve delusions, meta-analyses have generally integrated effects for positive symptoms rather than for delusions. Thus, it is still an open question whether CBTp is more effective with regard to change in delusions compared to treatment as usual (TAU) and to other interventions, and whether this effect remains stable over a follow-up period. Moreover, it would be interesting to explore whether newer studies that focus on specific factors involved in the formation and maintenance of delusions (causal-interventionist approach) are more effective than the first generation of CBTp studies. A systematic search of the trial literature identified 19 RCTs that compared CBTp with TAU and/or other interventions and reported delusions as an outcome measure. Meta-analytic integration resulted in a significant small to medium effect size for CBTp in comparison to TAU at end-of-therapy $(k=13 ; \bar{d}=0.27)$. However, the comparison between CBTp and TAU after an average follow-up period of 47 weeks was not statistically significant $(k=12, \bar{d}=0.16)$. When compared with other interventions, there was no significant effect of CBTp at end-of-therapy $(k=8 ; \bar{d}=0.16)$ and after a follow-up period $(k=5 ; \bar{d}=-0.04)$. Comparison between newer studies taking a causal-interventionist approach $(k=4)$ and first-generation studies showed a difference of 0.33 in mean effect sizes in favor of newer studies at end-of-therapy. The findings suggest that CBTp is superior to TAU post-therapy in bringing about a change in delusions, but that this change may not be maintained over the follow-up period. Moreover, interventions that focus on causal factors of delusions seem to be a promising approach to improving interventions for delusions.” 
Furthermore, the description of the included studies in the Results section was incorrect. Thus, a correction has been made the Results section, subsection Descriptive Information on Included Studies, paragraph two:

"Most studies ( $n=18)$ used observer-rated assessments of delusions such as the Psychotic Symptom Rating Scale $(k$ $=17$; PSYRATS: Haddock et al., 1999a) or the Maudsley Assessment of Delusions Scale $(k=1$; MADS: Wessely et al., 1993). Four of these studies did not use single-blind assessment (Tarrier et al., 1993; Foster et al., 2010; Kråkvik et al., 2013; Waller et al., 2015) and one study (Lincoln et al., 2012) used a self-report measure (Peters et al. Delusions Inventory: Peters et al., 1999). Most studies $(k=12)$ selectively included patients with delusions (Tarrier et al., 1993; Lewis et al., 2002; Durham et al., 2003; Valmaggia et al., 2005; O'Connor et al., 2007; Haddock et al., 2009; Foster et al., 2010; Kråkvik et al., 2013; Freeman et al., 2014, 2015; Morrison et al., 2014; Waller et al., 2015), but only one of these studies predefined change in delusions as the primary outcome (Waller et al., 2015).”

In addition, the mean effect size of the comparison between CBTp and TAU (and the corresponding statistics) after a followup period in the Results section is incorrect. Further, the reports on exclusion of studies with patients who did not use medication or suicidal patients, is incorrect. A correction has therefore been made to the Results section, subsection Comparison of CBTp and Treatment as usual (TAU), paragraph three:

"Results of comparisons of CBTp vs. TAU ( $k=12$ studies) after an average follow-up period of 47 weeks are depicted in Figure 4. The estimate for the mean effect size of CBTp compared to TAU was small and non-significant $(\bar{d}=0.16, S E=0.10$, $p=0.098, \mathrm{CI}:-0.03,0.35)$. The between-study variance was $\hat{\tau}^{2}=0.04$ (95\%-CI: $\left.0.00,0.23\right)$, and the $Q$-statistic $(Q=18.63$, $d f=11, p=0.068)$ was non-significant. The value of $I^{2}=$ $43.38 \%$ indicated a small to medium level of heterogeneity. The regression test for funnel plot asymmetry revealed a statistically non-significant result $(p=0.15)$, thus, there was no indication of a bias. Finally, we tested whether the results of both comparisons would change if we excluded two studies that assessed specific subpopulations: patients who did not use medication (Morrison et al., 2014) and suicidal patients (Tarrier et al., 2014). However, exclusion of these studies revealed comparable mean effect sizes (CBTp vs. TAU at end-of-treatment: $\bar{d}=0.32$; CBTp vs. TAU at follow-up: $\bar{d}=0.12)$."

Further, the results of the comparison between CBTp and TAU after a follow-up period were presented incorrectly in the Discussion section. A correction has therefore been made to the Discussion section, paragraph one:

"First, our results suggest that CBTp is more beneficial in changing delusions than standard treatment. However, the effect of CBTp on delusions did not remain stable after an average follow-up period of 47 weeks. Compared to other psychological interventions, CBTp did not prove to be better at changing delusions, neither at end-of-treatment, nor after a follow-up period. However, more recent studies that focused on factors that are hypothetically involved in the formation and maintenance of delusions rather than on the delusions per se, produced a numerically larger effect size of moderate magnitude compared to first-generation CBTp studies.”

An additional correction has been made to the Discussion section, paragraph two:

"With regard to comparisons between CBTp and standard treatment at end-of-therapy, our results are consistent with the large body of meta-analytic research which finds small to medium

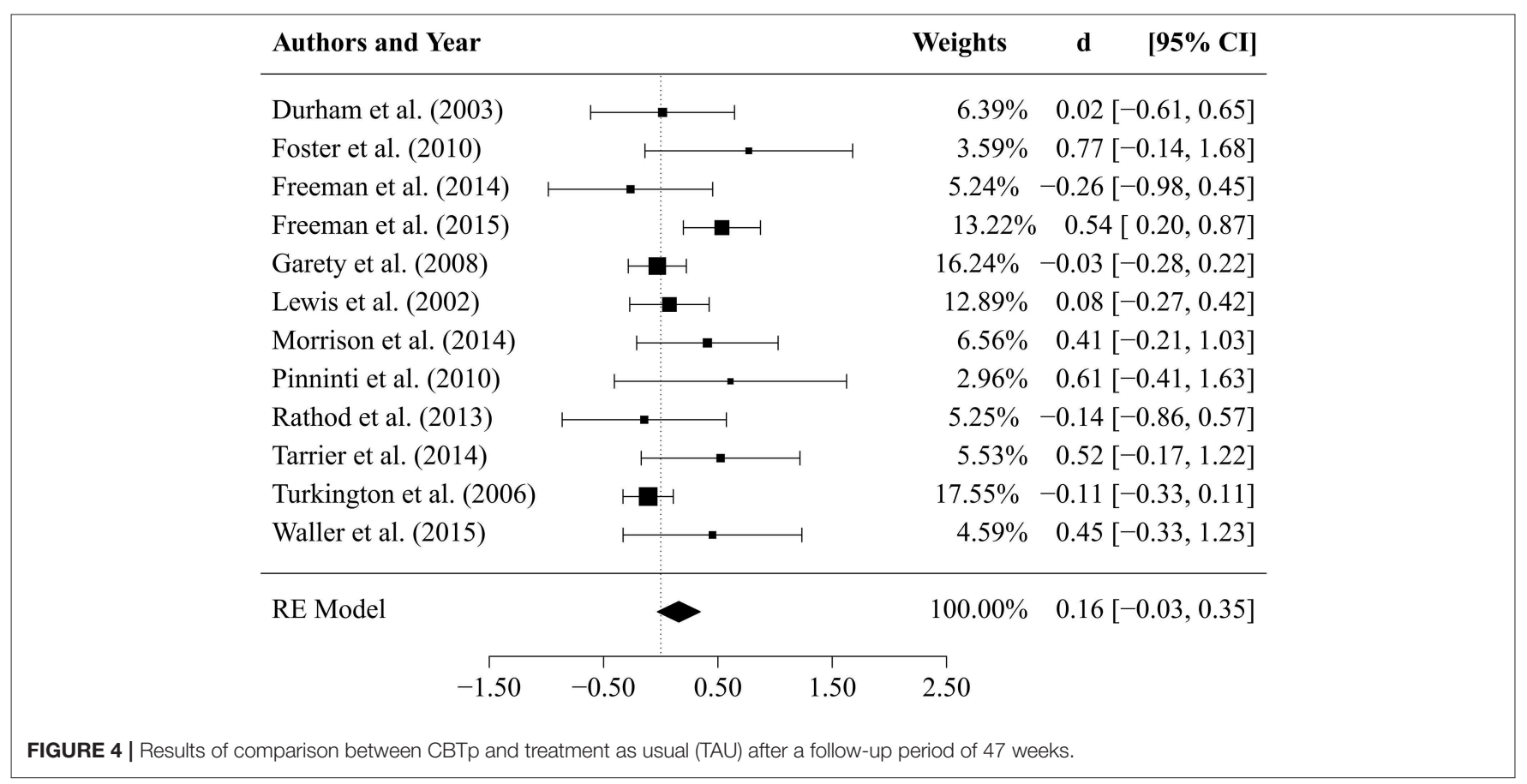


TABLE 1 | Studies included in the comparison of CBTp vs. TAU and CBTp vs. other psychological interventions: description of the intervention, patient characteristics and outcome measure.

\begin{tabular}{|c|c|c|c|c|c|c|c|c|c|c|}
\hline $\begin{array}{l}\text { Author and } \\
\text { Year }\end{array}$ & $\begin{array}{l}\text { Subject } \\
\text { characteristics: } \\
\text { Experimental } \\
\text { Condition (EC), } \\
\text { Control } \\
\text { Condition I (C1) } \\
\text { Control } \\
\text { Condition II } \\
\text { (CCII) }\end{array}$ & $\begin{array}{l}\text { Experimental } \\
\text { condition (EC) } \\
\text { CBT format } \\
\text { patients }\end{array}$ & $\begin{array}{l}\text { Control condition } \\
\text { I (CC I) format } \\
\text { patients }\end{array}$ & $\begin{array}{l}\text { Control condition } \\
\text { II (CC II) }\end{array}$ & $\begin{array}{l}\text { Duration of } \\
\text { intervention } \\
\mathrm{EC} / \mathrm{CCI} / \mathrm{CC} \text { II }\end{array}$ & $\begin{array}{l}\text { Total no. of } \\
\text { sessions, Mean } \\
\text { number of } \\
\text { sessions, EC/CC } \\
\text { I/CC II }\end{array}$ & $\begin{array}{l}\text { Selected } \\
\text { outcome } \\
\text { measure }\end{array}$ & $\begin{array}{l}\text { Blind } \\
\text { assessment? }\end{array}$ & $\begin{array}{l}\text { ITT- } \\
\text { data? }\end{array}$ & Follow-up \\
\hline $\begin{array}{l}\text { Cather et al., } \\
2005\end{array}$ & $\begin{array}{l}\text { Number of randomized } \\
\text { patients: } n=28, \\
\text { Diagnoses: } 17 \text { SZ; } 11 \\
\text { SA, Age: EC: } M=45.8 \\
\text { (SD=10.2) CCl: } M= \\
33.1 \text { (SD = 10.3), } \\
\text { Medication: EC: } \\
\text { 100\%/CCl: } 100 \%\end{array}$ & $\begin{array}{l}\text { Functional CBT, Based on } \\
\text { established manuals } \\
\text { (Kingdon and Turkington, } \\
\text { 1994; Fowler et al., 1995; } \\
\text { Chadwick et al., 1996; } \\
\text { Nelson, 1997), Number of } \\
\text { randomized patients: } n= \\
15\end{array}$ & $\begin{array}{l}\text { Psychoeducation, Number } \\
\text { of randomized patients: ( } n \\
=15 \text { ) }\end{array}$ & & 16/16 weeks & $\begin{array}{l}\text { Total number of } \\
\text { sessions: } 16 / 16^{6}\end{array}$ & PSYRATS del. & Yes & No & - \\
\hline $\begin{array}{l}\text { Durham et al., } \\
2003\end{array}$ & $\begin{array}{l}\text { Number of randomized } \\
\text { patients } n=66, \\
\text { Diagnoses: } 59 \text { SZ; } 5 \\
\text { SA; } 2 \text { DD, Age: EC: } M \\
=36 \text { (SD = 10.0)/CCl: } \\
M=36(S D=10.2) / C C \\
\text { II: } M=37 \text { (SD = 11.2), } \\
\text { Medication: EC: } \\
\text { 100\%/CC I: } 86 \%\end{array}$ & $\begin{array}{l}\text { CBT, Best practice based } \\
\text { on established manuals } \\
\text { (Tarrier, 1992; Kingdon and } \\
\text { Turkington, 1994), Number } \\
\text { of randomized patients: } n \\
=22\end{array}$ & $\begin{array}{l}\text { TAU, Number of } \\
\text { randomized patients: } n= \\
21\end{array}$ & $\begin{array}{l}\text { Supportive therapy, } \\
\text { Number of } \\
\text { randomized } \\
\text { patients: } n=23\end{array}$ & $\begin{array}{l}39 \text { weeks/-/22 } \\
\text { weeks }\end{array}$ & $\begin{array}{l}\text { Total number of } \\
\text { sessions: EC: } 20 /-/ C C \\
\text { II: } 20, \text { Mean number of } \\
\text { sessions: EC: } \\
\text { 14.8,/-/CC II: } 16.8, \\
\text { Dsessions }=-2.0\end{array}$ & PSYRATS del. & Yes & No & 52 weeks \\
\hline $\begin{array}{l}\text { Foster et al., } \\
2010\end{array}$ & $\begin{array}{l}\text { Number of randomized } \\
\text { patients } n=24 \text {, } \\
\text { Diagnoses: SZ, SA, and } \\
\text { DD } 1 \text {, Age: EC: } 40.0 \\
\text { (10.5)/CC I: } 39.1 \text { (9.2), } \\
\text { Medication: EC: } \\
\text { 92\%/CCl: } 83 \%\end{array}$ & $\begin{array}{l}\text { Worry-CBT, Fixed sessions } \\
\text { based on a manual (Wells, } \\
\text { 1997), Number of } \\
\text { randomized patients: } n= \\
12\end{array}$ & $\begin{array}{l}\text { TAU, Number of } \\
\text { randomized patients: } n= \\
12\end{array}$ & & 4 weeks/- & $\begin{array}{l}\text { Total number of } \\
\text { sessions: } 4 /-\end{array}$ & PSYRATS del. & No & No & 9 weeks \\
\hline $\begin{array}{l}\text { Freeman } \\
\text { et al., } 2015\end{array}$ & $\begin{array}{l}\text { Number of randomized } \\
\text { patients: } n=150, \\
\text { Diagnoses: } 111 \text { SZ; } 11 \\
\text { SA; } 10 \text { DD; } 18 \text { POS, } \\
\text { Age: EC: } 40.9(10.5) / C C \\
\text { I: } 42.1 \text { (13.1), } \\
\text { Medication: } 94 \%^{3}\end{array}$ & $\begin{array}{l}\text { Worry-CBT, Based on } \\
\text { self-help manual (Freeman } \\
\text { and Freeman, 2013), } \\
\text { Number of randomized } \\
\text { patients: } n=73\end{array}$ & $\begin{array}{l}\text { TAU, Number of } \\
\text { randomized patients: } n= \\
77\end{array}$ & - & 8 weeks./- & $\begin{array}{l}\text { Total number of } \\
\text { sessions: 6/-, Mean } \\
\text { number of sessions: } \\
\text { EC: } 5.5\end{array}$ & PSYRATS del. & Yes & No & 24 weeks \\
\hline $\begin{array}{l}\text { Freeman } \\
\text { et al., } 2014\end{array}$ & $\begin{array}{l}\text { Number of randomized } \\
\text { patients: } n=30, \\
\text { Diagnoses: } 22 \text { SZ; } 6 \\
\text { SA; } 1 \text { DD; } 1 \text { POS, Age: } \\
\text { EC: } 41.9 \text { (11.5)/CC I: } \\
41.5(13.1), \text { Medication: } \\
\text { EC: } 100 \% / C C \text { I: } 100 \%\end{array}$ & $\begin{array}{l}\text { Brief CBT, Based on } \\
\text { self-help manual (Freeman } \\
\text { and Freeman, 2012), } \\
\text { Number of randomized } \\
\text { patients: } n=15\end{array}$ & $\begin{array}{l}\text { TAU, Number of } \\
\text { randomized patients: } n= \\
15\end{array}$ & & 8 weeks/- & $\begin{array}{l}\text { Total number of } \\
\text { sessions: 6/-, Mean } \\
\text { number of session: EC: } \\
6.67 /-\end{array}$ & PSYRATS del. & Yes & No & 12 weeks \\
\hline
\end{tabular}




\begin{tabular}{|c|c|c|c|c|c|c|c|c|c|c|}
\hline $\begin{array}{l}\text { Author and } \\
\text { Year }\end{array}$ & $\begin{array}{l}\text { Subject } \\
\text { characteristics: } \\
\text { Experimental } \\
\text { Condition (EC), } \\
\text { Control } \\
\text { Condition I (C1) } \\
\text { Control } \\
\text { Condition II } \\
\text { (CCII) }\end{array}$ & $\begin{array}{l}\text { Experimental } \\
\text { condition (EC) } \\
\text { CBT format } \\
\text { patients }\end{array}$ & $\begin{array}{l}\text { Control condition } \\
\text { I (CC I) format } \\
\text { patients }\end{array}$ & $\begin{array}{l}\text { Control condition } \\
\text { II (CC II) }\end{array}$ & $\begin{array}{l}\text { Duration of } \\
\text { intervention } \\
\mathrm{EC} / \mathrm{CCI} / \mathrm{CC} \text { II }\end{array}$ & $\begin{array}{l}\text { Total no. of } \\
\text { sessions, Mean } \\
\text { number of } \\
\text { sessions, EC/CC } \\
\text { I/CC II }\end{array}$ & $\begin{array}{l}\text { Selected } \\
\text { outcome } \\
\text { measure }\end{array}$ & $\begin{array}{l}\text { Blind } \\
\text { assessment? }\end{array}$ & $\begin{array}{l}\text { ITT- } \\
\text { data? }\end{array}$ & Follow-up \\
\hline $\begin{array}{l}\text { Garety et al., } \\
2008\end{array}$ & $\begin{array}{l}\text { Number of randomized } \\
\text { patients: } n=328 \text {, } \\
\text { Diagnoses: } 258 \text { SZ; } 38 \\
\text { SA; } 5 \text { DD, Age: n.r., } \\
\text { Medication: n.r. }\end{array}$ & $\begin{array}{l}\text { CBT (carer + no-carer), } \\
\text { Based on an established } \\
\text { manual (Fowler et al., } \\
\text { 1995), Number of } \\
\text { randomized patients: } n= \\
160\end{array}$ & $\begin{array}{l}\text { TAU, Number of } \\
\text { randomized patients (carer } \\
+ \text { no-carer): } n=140\end{array}$ & $\begin{array}{l}\text { Family intervention, } \\
\text { Number of } \\
\text { randomized } \\
\text { patients: } n=28\end{array}$ & 39 weeks & $\begin{array}{l}\text { Total number of } \\
\text { sessions: } 20 /-, \text { Mean } \\
\text { number of sessions: } \\
\text { EC: } 14.3 /-/ C C \text { II: } 13.9 \text {, } \\
\text { Dessions }=0.4\end{array}$ & $\begin{array}{l}\text { PSYRATS del., } \\
\text { conviction and } \\
\text { delusion distress }\end{array}$ & Yes & No & 52 weeks \\
\hline $\begin{array}{l}\text { Haddock } \\
\text { et al., } 2009\end{array}$ & $\begin{array}{l}\text { Number of randomized } \\
\text { patients: } n=77 \text {, } \\
\text { Diagnoses: } 69 \text { SZ; } 7 \\
\text { SA; } 1 \text { POS, Age: EC: } \\
\text { 35.7 (12.5)/CC I: } 33.9 \\
\text { (9.7), Medication: EC: } \\
\text { 100\%/CC I: } 100 \%\end{array}$ & $\begin{array}{l}\text { CBT, Based on an } \\
\text { established manual } \\
\text { (Haddock et al., 2004), } \\
\text { Number of randomized } \\
\text { patients: } n=38\end{array}$ & $\begin{array}{l}\text { Social activity therapy, } \\
\text { Number of randomized } \\
\text { patients: } n=38\end{array}$ & & 26 weeks & $\begin{array}{l}\text { Total number of } \\
\text { sessions: } 25, \text { Mean } \\
\text { number of sessions: } \\
\text { EC: } 13.13 / C C \text { I: } 14.9 \text {, } \\
\text { D }_{\text {sessions }}=-1.77\end{array}$ & PSYRATS del. & Yes & No & 24 weeks \\
\hline $\begin{array}{l}\text { Kråkvik et al., } \\
2013\end{array}$ & $\begin{array}{l}\text { Number of randomized } \\
\text { patients: } n=55 \text {, } \\
\text { Diagnoses: } 34 \text { SZ/2 } \\
\text { SA/9 DD, Age: EC: } 37.5 \\
\text { (11.2)/ CC I: } 35.3 \text { (8.9), } \\
\text { Medication: EC: } \\
\text { 100\%/CC I: } 100 \%\end{array}$ & $\begin{array}{l}\text { CBT, Simplified version of } \\
\text { an established manual } \\
\text { (Chadwick et al., 1996), } \\
\text { Number of randomized } \\
\text { patients: } n=23\end{array}$ & $\begin{array}{l}\text { TAU }^{2}, \text { Number of } \\
\text { randomized patients: } n= \\
22\end{array}$ & - & 26 weeks & $\begin{array}{l}\text { Total number of } \\
\text { sessions: } 20\end{array}$ & $\begin{array}{l}\text { PSYRATS cognitive } \\
\text { and emotional }\end{array}$ & No & Yes & 52 weeks $^{2}$ \\
\hline $\begin{array}{l}\text { Lewis et al., } \\
2002\end{array}$ & 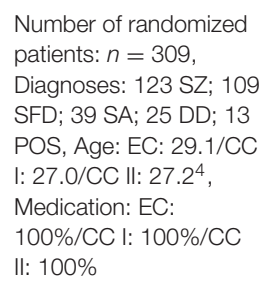 & $\begin{array}{l}\text { CBT, Based on an } \\
\text { established manual } \\
\text { (Haddock et al., 1999b), } \\
\text { Number of randomized } \\
\text { patients: } n=101\end{array}$ & $\begin{array}{l}\text { TAU, Number of } \\
\text { randomized patients: } n= \\
102\end{array}$ & $\begin{array}{l}\text { Supportive } \\
\text { counseling Number } \\
\text { of randomized } \\
\text { patients: } n=106\end{array}$ & 5 weeks & $\begin{array}{l}\text { Total number of } \\
\text { sessions: } 20, \text { Mean } \\
\text { number of sessions: } \\
\text { EC: } 16.1 /-/ C C \text { II: } 15.7 \text {, } \\
D_{\text {sessions }}=-0.4\end{array}$ & PSYRATS del. & Yes & No & 67 weeks \\
\hline $\begin{array}{l}\text { Lincoln et al., } \\
2012\end{array}$ & $\begin{array}{l}\text { Number of randomized } \\
\text { patients: } n=80 \text {, } \\
\text { Diagnoses: } 58 \text { SZ; } 13 \\
\text { SA; } 5 \text { DD; } 4 \text { APD, Age: } \\
\text { EC: } 33.2 \text { (10.4)/CC I: } \\
33.1 \text { (10.9), Medication: } \\
\text { EC: } 100 \% / C C \text { I: } 97 \%\end{array}$ & $\begin{array}{l}\text { CBTp, Based on an } \\
\text { established German } \\
\text { manual (Lincoln, 2006), } \\
\text { Number of randomized } \\
\text { patients: } n=40\end{array}$ & $\begin{array}{l}\mathrm{TAU}^{2}, \text { Number of } \\
\text { randomized patients: } n= \\
40\end{array}$ & - & 38 weeks & $\begin{array}{l}\text { No fixed number of } \\
\text { sessions. Mean number } \\
\text { of sessions EC: } 29 /-\end{array}$ & $\begin{array}{l}\text { PDI distress, } \\
\text { preoccupation, } \\
\text { conviction }\end{array}$ & No & Yes & 52 weeks $^{2}$ \\
\hline
\end{tabular}


TABLE 1 | Continued

\begin{tabular}{|c|c|c|c|c|c|c|c|c|c|c|}
\hline $\begin{array}{l}\text { Author and } \\
\text { Year }\end{array}$ & $\begin{array}{l}\text { Subject } \\
\text { characteristics: } \\
\text { Experimental } \\
\text { Condition (EC), } \\
\text { Control } \\
\text { Condition I (C1) } \\
\text { Control } \\
\text { Condition II } \\
\text { (CCII) }\end{array}$ & $\begin{array}{l}\text { Experimental } \\
\text { condition (EC) } \\
\text { CBT format } \\
\text { patients }\end{array}$ & $\begin{array}{l}\text { Control condition } \\
\text { I (CC I) format } \\
\text { patients }\end{array}$ & $\begin{array}{l}\text { Control condition } \\
\text { II (CC II) }\end{array}$ & $\begin{array}{l}\text { Duration of } \\
\text { intervention } \\
\mathrm{EC} / \mathrm{CCI} / \mathrm{CC} \text { II }\end{array}$ & $\begin{array}{l}\text { Total no. of } \\
\text { sessions, Mean } \\
\text { number of } \\
\text { sessions, EC/CC } \\
\text { I/CC II }\end{array}$ & $\begin{array}{l}\text { Selected } \\
\text { outcome } \\
\text { measure }\end{array}$ & $\begin{array}{l}\text { Blind } \\
\text { assessment? }\end{array}$ & $\begin{array}{l}\text { ITT- } \\
\text { data? }\end{array}$ & Follow-up \\
\hline $\begin{array}{l}\text { Morrison } \\
\text { et al., } 2014\end{array}$ & $\begin{array}{l}\text { Number of randomized } \\
\text { patients: } n=74 \text {, } \\
\text { Diagnoses: SZ, SA, and } \\
\text { DD }, \text { Age: EC: } 33.0 \\
\text { (13.1)/CC I: } 29.7(11.9) \text {, } \\
\text { Medication: EC: } 0 \% / C C \\
\text { I: } 0 \%\end{array}$ & $\begin{array}{l}\text { CBTp, Based on } \\
\text { established manuals } \\
\text { (Morrison et al., 2004; } \\
\text { Kingdon and Turkington, } \\
\text { 2005), Number of } \\
\text { randomized patients: } n= \\
37\end{array}$ & $\begin{array}{l}\text { TAU, Number of } \\
\text { randomized patients: } n= \\
37\end{array}$ & - & 39 weeks & $\begin{array}{l}\text { Total number of } \\
\text { sessions: } 26, \text { Mean } \\
\text { number of sessions: } \\
\text { EC: } 13.3 /-\end{array}$ & $\begin{array}{l}\text { PSYRATS cognitive } \\
\text { and emotional }\end{array}$ & Yes & No & 19 weeks \\
\hline $\begin{array}{l}\text { O'Connor } \\
\text { et al., } 2007\end{array}$ & $\begin{array}{l}\text { Number of randomized } \\
\text { patients: } n=24 \text {, } \\
\text { Diagnoses: } 24 \text { DD, Age: } \\
\text { EC: } 40.0 \text { (9.4)/CC I: } \\
\text { 36.8 (13.5), Medication: } \\
\text { EC: } 100 \% / C C \text { I: } 100 \%\end{array}$ & $\begin{array}{l}\text { CBTp, Based on } \\
\text { established manuals } \\
\text { : (Fowler et al., 1995; } \\
\text { Chadwick et al., 1996), } \\
\text { Number of randomized } \\
\text { patients: } n=12\end{array}$ & $\begin{array}{l}\text { Attention placebo control, } \\
\text { Number of randomized } \\
\text { patients: } n=12\end{array}$ & - & 24 weeks & $\begin{array}{l}\text { Total number of } \\
\text { sessions: } 24\end{array}$ & MADS & Yes & No & - \\
\hline $\begin{array}{l}\text { Pinninti et al., } \\
2010\end{array}$ & $\begin{array}{l}\text { Number of randomized } \\
\text { patients: } n=33 \text {, } \\
\text { Diagnoses: } 11 \mathrm{SZ} ; 22 \\
\text { SA, Age: } 40.0(11.0)^{3} \text {, } \\
\text { Medication: EC: } \\
\text { 100\%/CC I: } 100 \%\end{array}$ & $\begin{array}{l}\text { CBTp, Not manualized, } \\
\text { Number of randomized } \\
\text { patients: } n=18\end{array}$ & $\begin{array}{l}\text { TAU, Number of } \\
\text { randomized patients: } n= \\
15\end{array}$ & - & 12 weeks & $\begin{array}{l}\text { Total number of } \\
\text { sessions: } 12 \text {, Mean } \\
\text { number of sessions EC: } \\
11.9 /-\end{array}$ & PSYRATS del. & Yes & No & 24 weeks \\
\hline $\begin{array}{l}\text { Rathod et al., } \\
2013\end{array}$ & $\begin{array}{l}\text { Number of randomized } \\
\text { patients } n=35 \text {, } \\
\text { Diagnoses: SZ, SA, and } \\
\text { DD }{ }^{1}, \text { Age: EC: } 31.4 \\
\text { (12.3)/CC I: } 35.6(10.7) \text {, } \\
\text { Medication: EC: } \\
\text { 100\%/CC I: } 100 \%\end{array}$ & $\begin{array}{l}\text { Culturally adapted CBTp } \\
\text { Based on a study protocol } \\
\text { (Rathod et al., 2010), } \\
\text { Number of randomized } \\
\text { patients: } n=17\end{array}$ & $\begin{array}{l}\text { TAU, Number of } \\
\text { randomized patients: } n= \\
15\end{array}$ & - & 18 weeks & $\begin{array}{l}\text { Total number of } \\
\text { sessions: } 16 \text {, Mean } \\
\text { number of sessions: } \\
\text { EC: } 13.6 /-\end{array}$ & CPRS del. & Yes & Yes & 26 weeks \\
\hline $\begin{array}{l}\text { Tarrier et al., } \\
1993\end{array}$ & $\begin{array}{l}\text { Number of randomized } \\
\text { patients: } n=27 \text {, } \\
\text { Diagnoses: } 307 \text { SZ, } \\
\text { Age: EC: } 42.8(12.3) / C C \\
\text { I: } 42.8 \text { (12.3), } \\
\text { Medication: } E \text { C: } \\
\text { 100\%/CC I: } 100 \%\end{array}$ & $\begin{array}{l}\text { Coping strategy } \\
\text { enhancement, Based on } \\
\text { an established manual } \\
\text { (Tarrier, 1992), Number of } \\
\text { randomized patients: } n= \\
15\end{array}$ & $\begin{array}{l}\text { Problem solving, Number } \\
\text { of randomized patients: } n \\
=12\end{array}$ & - & 5 weeks & $\begin{array}{l}\text { Total number of } \\
\text { sessions: } 10\end{array}$ & PAS delusions & No & No & 31 weeks \\
\hline
\end{tabular}


TABLE 1 | Continued

\begin{tabular}{|c|c|c|c|c|c|c|c|c|c|c|}
\hline $\begin{array}{l}\text { Author and } \\
\text { Year }\end{array}$ & $\begin{array}{l}\text { Subject } \\
\text { characteristics: } \\
\text { Experimental } \\
\text { Condition (EC), } \\
\text { Control } \\
\text { Condition I (C1) } \\
\text { Control } \\
\text { Condition II } \\
\text { (CCII) }\end{array}$ & $\begin{array}{l}\text { Experimental } \\
\text { condition (EC) } \\
\text { CBT format } \\
\text { patients }\end{array}$ & $\begin{array}{l}\text { Control condition } \\
\text { I (CC I) format } \\
\text { patients }\end{array}$ & $\begin{array}{l}\text { Control condition } \\
\text { II (CC II) }\end{array}$ & $\begin{array}{l}\text { Duration of } \\
\text { intervention } \\
\mathrm{EC} / \mathrm{CCI} / \mathrm{CC} \text { II }\end{array}$ & $\begin{array}{l}\text { Total no. of } \\
\text { sessions, Mean } \\
\text { number of } \\
\text { sessions, EC/CC } \\
\text { I/CC II }\end{array}$ & $\begin{array}{l}\text { Selected } \\
\text { outcome } \\
\text { measure }\end{array}$ & $\begin{array}{l}\text { Blind } \\
\text { assessment? }\end{array}$ & $\begin{array}{l}\text { ITT- } \\
\text { data? }\end{array}$ & Follow-up \\
\hline $\begin{array}{l}\text { Tarrier et al., } \\
2014\end{array}$ & $\begin{array}{l}\text { Number of randomized } \\
\text { patients } n=49 \text {, } \\
\text { Diagnoses: SZ, SA, DD, } \\
\text { POS }{ }^{1}, \text { Age: EC: } 32.6 \\
\text { (11.7)/CC I: } 37.3 \text { (14.2), } \\
\text { Medication: EC: } \\
\text { 100\%/CC I: } 100 \%\end{array}$ & $\begin{array}{l}\text { CBT for suicidal patients, } \\
\text { Based on a manual (Tarrier } \\
\text { et al., 2013), Number of } \\
\text { randomized patients: } n= \\
25\end{array}$ & $\begin{array}{l}\text { TAU, Number of } \\
\text { randomized patients: } n= \\
24\end{array}$ & - & 12 weeks & $\begin{array}{l}\text { Total number of } \\
\text { sessions: } 24\end{array}$ & PSYRATS del. & Yes & No & 17 weeks \\
\hline $\begin{array}{l}\text { Turkington } \\
\text { et al., } 2006\end{array}$ & $\begin{array}{l}\text { Number of randomized } \\
\text { patients: } n=422 \text {, } \\
\text { Diagnoses: } 422 \text { SZ, } \\
\text { Age: } n \text {. r., Medication: } \\
\text { EC: } 100 \% / C C \text { I: } 100 \%\end{array}$ & $\begin{array}{l}\text { CBTp, Based on } \\
\text { established manuals } \\
\text { (Kingdon and Turkington, } \\
\text { 1994, 2005), Number of } \\
\text { randomized patients: } n= \\
281\end{array}$ & $\begin{array}{l}\mathrm{TAU}^{2}, \text { Number of } \\
\text { randomized patients: } n= \\
141\end{array}$ & - & 10.5 weeks & $\begin{array}{l}\text { Total number of } \\
\text { sessions: Mean number } \\
\text { of sessions: EC: 6/- }\end{array}$ & PSYRATS del. & Yes & No & 52 weeks \\
\hline $\begin{array}{l}\text { Valmaggia } \\
\text { et al., } 2005\end{array}$ & $\begin{array}{l}\text { Number of randomized } \\
\text { patients: } n=62 \text {, } \\
\text { Diagnoses: } 62 \text { SZ, Age: } \\
\text { EC: } 35.4 \text { (10.5)/CC I: } \\
35.5 \text { (11.4), Medication: } \\
\text { EC: } 100 \% / C C \text { I: } 100 \%\end{array}$ & $\begin{array}{l}\text { CBTp, Based on an } \\
\text { established manual } \\
\text { (Kingdon and Turkington, } \\
\text { 1994), Number of } \\
\text { randomized patients: } n= \\
36\end{array}$ & $\begin{array}{l}\text { Supportive counseling, } \\
\text { Number of randomized } \\
\text { patients: } n=26\end{array}$ & - & 22 weeks & $\begin{array}{l}\text { Total number of } \\
\text { sessions: } 16\end{array}$ & $\begin{array}{l}\text { PSYRATS cognitive } \\
\text { and emotional } \\
\text { scale }\end{array}$ & e Yes & Yes & 48 weeks \\
\hline $\begin{array}{l}\text { Waller et al., } \\
2015\end{array}$ & $\begin{array}{l}\text { Number of randomized } \\
\text { patients: } n=31 \\
\text { Diagnoses: } 27 \mathrm{SZ}, 2 \\
\text { SA, } 2 \text { DD, Age: EC: } \\
\text { 39.1 (10.5)/CC I: } 43.0 \\
\text { (10.7), Medication: EC: } \\
\text { 90\%/CC I: } 91 \%\end{array}$ & $\begin{array}{l}\text { Focused CBT, Sessions } \\
\text { described in the study, } \\
\text { Number of randomized } \\
\text { patients: } n=20\end{array}$ & $\begin{array}{l}\text { TAU, Number of } \\
\text { randomized patients: } n= \\
11\end{array}$ & - & 5 weeks & $\begin{array}{l}\text { Total number of } \\
\text { sessions: } 4\end{array}$ & PSYRATS del. & No & Yes & 8 weeks \\
\hline
\end{tabular}

M, Mean; SD, Standard deviation; TAU, Treatment as Usual; EC, Experimental condition; CCI, Control condition I; CCII, Control condition II; SZ, Schizophrenia; SA, Schizoaffective Disorder; DD, Delusional disorder; APD, Acute psychotic disorder: POS, Psychosis not otherwise specified; SFD. Schizophreniform disorder:Medication, percentage of patients treated with antipsychotic medication. PSYRATSdel., PSYRATS delusions score; CPRS, Comprehensive Psychopathology Rating Scale; PAS, Psychiatric Assessment Scale;n.r., not reported;Dsessions, Mean number of CBT sessions-Mean number of other therapy sessions; ${ }^{1}$ no information on diagnosis ratio; ${ }^{2}$ study was not included in follow-up comparison between CBTp and TAU, as the study used a wait-list design and comparisons between CBTp and TAU are not possible at follow-up assessment; ${ }^{3}$ variable was only reported for all patients; ${ }^{4}$ SD was not reported. 
effect sizes for positive symptoms (Lincoln et al., 2008; Wykes et al., 2008; Sarin et al., 2011; Jauhar et al., 2014). Moreover, our results are comparable with the recent meta-analysis by van der Gaag et al. (2014) that focused on change in delusions in individually-tailored formulation-based CBTp. However, they reported a slightly higher estimated effect size $(k=9 ; \bar{d}=$ $0.36,95 \%$-CI: $0.08,0.63)$ which seems to be the result of using a smaller pool of studies. The broader selection of studies in our meta-analysis produced a slightly smaller effect size; this effect size had a smaller confidence interval $(\bar{d}=0.27,95 \%$-CI: $0.08,0.47)$. Thus, the broader inclusion criteria we used lead to a slightly smaller, but also to a more precise estimation of the mean effect size of change in delusions at end-of-therapy. Nevertheless, we also investigated the stability of the effects, but CBTp was not more effective than standard treatment over an average follow-up period of 47 weeks. Due to the small number of RCTs that addressed both the question of change in delusions and the stability of CBTp over a follow-up period, more studies are needed to be able to draw more definite conclusions in regard to long-term effects."

A correction has also been made to the Discussion section, paragraph three:

"It is important to note that we found a small to medium amount of variance that is due to the heterogeneity between the studies (about 42\%). This variance is largely due to the study by Kråkvik et al. (2013). This study included patients with both auditory hallucinations and delusions and produced a quite large effect size $(\bar{d}=0.94)$, which might have been influenced by the lack of blinding."

A correction has also been made to the Discussion section, paragraph ten:

\section{REFERENCES}

Cather, C., Penn, D., Otto, M. W., Yovel, I., Mueser, K. T., and Goff, D. C. (2005). A pilot study of functional Cognitive Behavioral Therapy (fCBT) for schizophrenia. Schizophr. Res. 74, 201-209. doi: 10.1016/j.schres.2004. 05.002

Chadwick, P., Birchwood, M. J., and Trower, P. (1996). Cognitive Behaviour Therapy for Delusions, Voices and Paranoia. Chichester: Wiley.

Durham, R. C., Guthrie, M., Morton, R. V., Reid, D. A., Treliving, L. R., Fowler, D., et al. (2003). Tayside-Fife clinical trial of cognitive-behavioural therapy for medication-resistant psychotic symptoms. Results to 3-month follow-up. Br. J. Psychiatry 182, 303-311. doi: 10.1192/bjp.182.4.303

Foster, C., Startup, H., Potts, L., and Freeman, D. (2010). A randomised controlled trial of a worry intervention for individuals with persistent persecutory delusions. J. Behav. Ther. Exp. Psychiatry 41, 45-51. doi: 10.1016/j.jbtep.2009.09.001

Fowler, D., Garety, P. A., and Kuipers, E. (1995). Cognitive Behaviour Therapy for Psychosis. Theory and Practice. Chichester: Wiley.

Freeman, D., Dunn, G., Startup, H., Pugh, K., Cordwell, J., Mander, H., et al. (2015). Effects of cognitive behaviour therapy for worry on persecutory delusions in patients with psychosis (WIT): a parallel, single-blind, randomised controlled trial with a mediation analysis. Lancet Psychiatry 2, 305-313. doi: 10.1016/s2215-0366(15)00039-5

Freeman, D., and Freeman, J. (2012). You Can Be Happy: The Scientifical Proven Way to Change How You Feel. Harlow: Prentice Hall Life.

Freeman, D., and Freeman, J. (2013). How to Keep Calm and Carry On. Harlow: Pearson.
"To sum up, our results suggest that CBTp is superior to TAU in regard to changing delusions, but CBTp effects might not be maintained over the course of the follow-up period. Moreover, at present, CBTp is not superior to other effective interventions, neither at end-of-therapy nor after a follow-up period. Finally, interventions that focus specifically on cognitive and emotional factors that are hypothetically involved in the formation and maintenance of delusions seem to be slightly more effective and thus are a promising approach to improving interventions for delusions."

Furthermore, the effect size of the Turkington study (Turkington et al., 2006) was incorrect. The correct effect size is: $\bar{d}=-0.11(-0.33,0.11)$. Furthermore, the mean effect size of the last row of the RE Model is incorrect. The correct effect size is: $\bar{d}$ $=0.16(-0.03,0.35)$.

Thus, Figure 4 has been corrected.

Furthermore, in Table 1, four studies (Tarrier et al., 1993; Lincoln et al., 2012; Kråkvik et al., 2013 and Waller et al., 2015) were incorrectly described as studies with blind assessment and their blinding status should be described as non-blinded ("No"). Further, one study ('O'Connnor) was incorrectly described as non-blind and its blinding status should be described as blinded ("Yes").

Thus Table 1 has been corrected.

The authors apologize for these errors and would like to thank Prof. Dr. Laws for pointing them out (Laws, 2016). Unfortunately, we did not receive notice of the comment by Prof. Dr. Laws until this year (2019). In response, we have corrected the errors, revised the discussion of the article and changed the scientific conclusions that were influenced by these errors. The original article has been updated.

Freeman, D., Pugh, K., Dunn, G., Evans, N., Sheaves, B., Waite, F., et al. (2014). An early Phase II randomised controlled trial testing the effect on persecutory delusions of using CBT to reduce negative cognitions about the self: the potential benefits of enhancing self confidence. Schizophr. Res. 160, 186-192. doi: 10.1016/j.schres.2014.10.038

Garety, P. A., Fowler, D. G., Freeman, D., Bebbington, P., Dunn, G., and Kuipers, E. (2008). Cognitive-behavioural therapy and family intervention for relapse prevention and symptom reduction in psychosis: randomised controlled trial. Br. J. Psychiatry 192, 412-423. doi: 10.1192/bjp.bp.107.043570

Haddock, G., Barrowclough, C., Shaw, J. J., Dunn, G., Novaco, R. W., and Tarrier, N. (2009). Cognitive-behavioural therapy v. social activity therapy for people with psychosis and a history of violence: randomised controlled trial. Br. J. Psychiatry 194, 152-157. doi: 10.1192/bjp.bp.107.039859

Haddock, G., Lowens, I., Brosnan, N., Barrowclough, C., and Novaco, R. W. (2004). Cognitive behaviour therapy for inpatients with psychosis and anger problems within a low secure environment. Behav. Cogn. Psychother. 32, 77-98. doi: 10.1017/S1352465804001055

Haddock, G., McCarron, J., Tarrier, N., and Faragher, E. B. (1999a). Scales to measure dimensions of hallucinations and delusions: the psychotic symptom rating scales (PSYRATS). Psychol. Med. 29, 879-889. doi: 10.1017/S0033291799008661

Haddock, G., Tarrier, N., Morrison, A. P., Hopkins, R., Drake, R., and Lewis, S. (1999b). A pilot study evaulating the effectiveness of individual inpatient cognitive-behavioural therapy in early psychosis. Sociol. Psychiatry Psychiatr. Epidemiol. 34, 254-258. doi: 10.1007/s001270050141

Jauhar, S., McKenna, P. J., Radua, J., Fung, E., Salvador, R., and Laws, K. R. (2014). Cognitive-behavioural therapy for the symptoms of schizophrenia: systematic 
review and meta-analysis with examination of potential bias. $\mathrm{Br}$. J. Psychiatry 204, 20-29. doi: 10.1192/bjp.bp.112.116285

Kingdon, D. G., and Turkington, D. (1994). Cognitive-behavioural Therapy of Schizophrenia. New York, NY: Guilford Press.

Kingdon, D. G., and Turkington, D. (2005). Cognitive-behavioural Therapy of Schizophrenia. New York, NY: Guilford Press.

Kråkvik, B., Gråwe, R. W., Hagen, R., and Stiles, T. C. (2013). Cognitive behaviour therapy for psychotic symptoms: a randomized controlled effectiveness trial. Behav. Cogn. Psychother. 41, 511-524. doi: 10.1017/S1352465813 000258

Laws, K. R. (2016). Commentary: Does Cognitive Behavior Therapy for psychotic disorders (CBTp) show a sustainable effect on delusions? A meta-analysis. Front. Psychol. 7:59. doi: 10.3389/fpsyg.2016.00059

Lewis, S., Tarrier, N., Haddock, G., Bentall, R., Kinderman, P., Kingdon, D., et al. (2002). Randomised controlled trial of cognitive-behavioural therapy in early schizophrenia: acute-phase outcomes. Br. J. Psychiatry Suppl. 43, s91-s97. doi: 10.1192/bjp.181.43.s91

Lincoln, T. M. (2006). Kognitive Verhaltenstherapie der Schizophrenie. Göttingen: Hogrefe.

Lincoln, T. M., Suttner, C., and Nestoriuc, Y. (2008). Wirksamkeit kognitiver Interventionen für Schizophrenie. Psychol. Rundschau 59, 217-232. doi: 10.1026/0033-3042.59.4.217

Lincoln, T. M., Ziegler, M., Mehl, S., Kesting, M. L., Lüllmann, E., Westermann, S., et al. (2012). Moving from efficacy to effectiveness in cognitive behavioral therapy for psychosis: a randomized clinical practice trial. J. Consult. Clin. Psychol. 80, 674-686. doi: 10.1037/a0028665

Morrison, A., Renton, J., Dunn, H., Williams, S., and Bentall, R. (2004). Cognitive Therapy for Psychosis: A Formulation-Based Approach. New York, NY: Routledge.

Morrison, A. P., Turkington, D., Pyle, M., Spencer, H., Brabban, A., Dunn, G., et al. (2014). Cognitive therapy for people with schizophrenia spectrum disorders not taking antipsychotic drugs: a single-blind randomised controlled trial. Lancet 383, 1395-1403. doi: 10.1016/S0140-6736(13)62246-1

Nelson, H. E. (1997). Cognitive Behavioural Therapy with Schizophrenia. A Practice Manual. Cheltenham: Stanley Thornes Publishers.

O'Connor, K., Stip, E., Pélissier, M. C., Aardema, F., Guay, S., Gaudette, G., et al. (2007). Treating delusional disorder: a comparison of cognitive-behavioural therapy and attention placebo control. Can. J. Psychiatry 52, 182-190.

Peters, E. R., Joseph, S. A., and Garety, P. A. (1999). Measurement of delusional ideation in the normal population: introducing the PDI (Peters et al., Delusions Inventory). Schizophr. Bull. 25, 553-576. doi: 10.1093/oxfordjournals.schbul.a033401

Pinninti, N. R., Rissmiller, D. J., and Steer, R. A. (2010). Cognitive-behavioral therapy as an adjunct to second-generation antipsychotics in the treatment of schizophrenia. Psychiatr. Serv. 61, 940-943. doi: 10.1176/ps.2010.61.9.940

Rathod, S., Kingdon, D., Phiri, P., and Gobbi, M. (2010). Developing culturally sensitive cognitive behaviour therapy for psychosis for ethnic minority groups by exploration and incorporation of Service Users and Health Professionals views and opinions. Behav. Cogn. Psychother. 38, 511-533. doi: $10.1017 /$ S1352465810000378

Rathod, S., Phiri, P., Harris, S., Underwood, C., Thagadur, M., Padmanabi, U., et al. (2013). Cognitive behaviour therapy for psychosis can be adapted for minority ethnic groups: a randomised controlled trial. Schizophr. Res. 143, 319-326. doi: 10.1016/j.schres.2012.11.007
Sarin, F., Wallin, L., and Widerlöv, B. (2011). Cognitive behavior therapy for schizophrenia: a meta-analytical review of randomized controlled trials. Nord. J. Psychiatry 65, 162-174. doi: 10.3109/08039488.2011. 577188

Tarrier, N. (1992). "Management and modification of residual psychotic symptoms," in Innovations in the Psychological Management of Schizophrenia, eds M. Birchwood and N. Tarrier (Chichester: Wiley), 147-170.

Tarrier, N., Beckett, R., Harwood, S., Baker, A., Yusupoff, L., and Ugarteburu, I. (1993). A trial of two cognitive-behavioural methods of treating drug-resistant residual psychotic symptoms in Schizophrenic patients: I. Outcome. Br. J. Psychiatry 162, 524-532. doi: 10.1192/bjp.162.4.524

Tarrier, N., Gooding, P., Pratt, D., Kelly, J., Awenat, Y., and Maxwell, J. (2013). Cognitive Behavioural Prevention of Suicide in Psychosis: A Treatment Manual. London: Routledge.

Tarrier, N., Kelly, J., Maqsood, S., Snelson, N., Maxwell, J., Law, H., et al. (2014). The cognitive behavioural prevention of suicide in psychosis: a clinical trial. Schizophr. Res. 156, 204-210. doi: 10.1016/j.schres.201 4.04 .029

Turkington, D., Kingdon, D., Rathod, S., Hammond, K., Pelton, J., and Mehta, R. (2006). Outcomes of an effectiveness trial of cognitive-behavioural intervention by mental health nurses in schizophrenia. Br. J. Psychiatry 189, 36-40. doi: 10.1192/bjp.bp.105.010884

Valmaggia, L. R., van der Gaag, M., Tarrier, N., Pijnenborg, M., and Slooff, C. J. (2005). Cognitive-behavioural therapy for refractory psychotic symptoms of schizophrenia resistant to atypical antipsychotic medication. Randomised controlled trial. Br. J. Psychiatry 186, 324-330. doi: 10.1192/bjp.1 86.4.324

van der Gaag, M., Valmaggia, L. R., and Smit, F. (2014). The effects of individually tailored formulation-based cognitive behavioural therapy in auditory hallucinations and delusions: a metaanalysis. Schizophr. Res. 156, 30-37. doi: 10.1016/j.schres.2014. 03.016

Waller, H., Emsley, R., Freeman, D., Bebbington, P., Dunn, G., Fowler, D., et al. (2015). Thinking well: a randomised controlled feasibility study of a new CBT therapy targeting reasoning biases in people with distressing persecutory delusional beliefs. J. Behav. Ther. Exp. Psychiatry 48, 82-89. doi: 10.1016/j.jbtep.2015.02.007

Wells, A. (1997). Cognitive Therapy of Anxiety Disorders: A Practice Manual and Conceptual Guide. Chichester: Wiley.

Wessely, S., Buchanan, A., Reed, A., Cutting, J., Everitt, B., Garety, P., et al. (1993). Acting on delusions I: prevalence. Br. J. Psychiatry 163, 69-76. doi: 10.1192/bjp.163.1.69

Wykes, T., Steel, C., Everitt, B., and Tarrier, N. (2008). Cognitive behavior therapy for schizophrenia: effect sizes, clinical models, and methodological rigor. Schizophr. Bull. 34, 523-537. doi: 10.1093/schbul/ sbm 114

Copyright (c) 2019 Mehl, Werner and Lincoln. This is an open-access article distributed under the terms of the Creative Commons Attribution License (CC BY). The use, distribution or reproduction in other forums is permitted, provided the original author(s) and the copyright owner(s) are credited and that the original publication in this journal is cited, in accordance with accepted academic practice. No use, distribution or reproduction is permitted which does not comply with these terms. 